\title{
Long-term effectiveness of plasma-derived hepatitis $B$ vaccine 22-28 years after immunization in a hepatitis $B$ virus endemic rural area: is an adult booster dose needed?
}

\author{
H. LI ${ }^{1,2}$, G. J. LI ${ }^{1,2}$, Q. Y. CHEN ${ }^{1,2}$, Z. L. FANG ${ }^{1,2}$, X. Y. WANG ${ }^{1,2}$, C. TAN $^{1,2}$, \\ Q. L. YANG ${ }^{1,2}$, F. Z. WANG ${ }^{3}$, F. WANG ${ }^{4}$, S. ZHANG ${ }^{4}$, S. L. BI ${ }^{4}$ AND L. P. SHEN ${ }^{4}$ \\ ${ }^{1}$ Laboratory for the Control and Prevention of Viral Hepatitis, Guangxi Center for Disease Control and \\ Prevention, Nanning, China \\ ${ }^{2}$ Guangxi Key Laboratory for the Control and Prevention of Viral Hepatitis, Nanning, China \\ ${ }^{3}$ National Immunization Program, Chinese Center for Disease Control and Prevention, Beijing, China \\ ${ }^{4}$ National Institute for Viral Disease Control and Prevention, Chinese Center for Disease Control and Prevention, \\ Beijing, China
}

Received 26 July 2016; Final revision 12 November 2016; Accepted 23 November 2016; first published online 9 January 2017

\section{SUMMARY}

Longan County is considered a highly endemic area for hepatitis B virus (HBV). The plasmaderived vaccine has been used in newborns in this area since 1987. A cross-sectional survey was conducted to evaluate the long-term effectiveness of this vaccine. In total, 1634 participants born during 1987-1993 and who had received a series of plasma-derived HB vaccinations at ages 0,1 , and 6 months were enrolled. Serological HBV markers were detected and compared with previous survey data. Overall the prevalence of hepatitis B surface antigen (HBsAg) in all participants was $3.79 \% ; 3.47 \%$ of subjects who had received the first dose within $24 \mathrm{~h}$ were HBsAg positive, and $8.41 \%$ of subjects who had received a delayed first dose were also HBsAg positive. There were 1527 subjects identified who had received the first dose within $24 \mathrm{~h}$ and whose $\mathrm{HBsAg}$ and anti-HBc prevalence increased yearly after immunization, while the anti-HBspositive rate and vaccine effectiveness declined. The geometric mean concentration of antibody in the anti-HB-positive participants was $55.13 \mathrm{mIU} / \mathrm{ml}$ and this declined after immunization. Fewer than $2 \cdot 0 \%$ of participants had anti-HB levels $\geqslant 1000 \mathrm{mIU} / \mathrm{ml}$. The data show that the protective efficacy of the plasma-derived vaccinations declined and administration of $\mathrm{HB}$ vaccine within $24 \mathrm{~h}$ of birth was very important. To reduce the risk of HBV infection in this highly endemic area, a booster dose might be necessary if anti-HBs levels fall below $10 \mathrm{mIU} / \mathrm{ml}$ after age 18 years.

Furthermore, studies on the immune memory induced by plasma-derived HB vaccine are needed.

Key words: Hepatitis B virus, long-term effectiveness, plasma-derived hepatitis B vaccine, primary immunization.

\section{INTRODUCTION}

Hepatitis B virus (HBV) infection is a public health problem of major concern worldwide. It is estimated

\footnotetext{
* Author for correspondence: Dr L. P. Shen, National Institute for Viral Disease Control and Prevention, Chinese Center for Disease Control and Prevention, Beijing, China. (Email:slpss128@126.com)
}

that 240 million people are chronically infected with HBV, and about 780000 people die of hepatitis B infection annually - 650000 from cirrhosis and liver cancer and 130000 from acute HB [1]. Immunization is the most effective method to control and prevent $\mathrm{HBV}$ infection and its serious long-term consequences. According to the World Health Organization, universal immunization has been 
implemented in 168 countries and has an outstanding record of safety and efficacy [2]. The effective implementation of HBV immunization has resulted in substantial decreases in disease burden, carriage rates, and HB-related morbidity and mortality [2-6]. After implementation of HBV immunization in China, the prevalence of hepatitis B surface antigen (HBsAg) fell from $10 \cdot 3 \%$ in 1980 to $9 \cdot 8 \%$ in 1992 and $7 \cdot 2 \%$ in 2006 [7] A recent study showed that the decline in $\mathrm{HBsAg}$ prevalence in all age groups in China was strongly associated with increased vaccine coverage [8]. Guangxi was one of the provinces that had a high HBV prevalence estimated in 1980 [9], but by 2006 the prevalence had decreased to $8 \cdot 3 \%$ [10].

Longan County is a remote, undeveloped district in rural Guangxi province. In 1985, it was an HBV-endemic area with an HBsAg prevalence of $16.9 \%$ in those aged between 1 and 60 years [11]. After the completion of baseline surveys on the prevalence of HBV infection in 1985, large-scale HBV immunization was undertaken in five areas of China: Longan in Guangxi province, Zhengding in Hebei province, Xiangtan in Hunan province, Tongde in Qinhai province, and Shanghai city [12]. From 1987 to 1996 , newborns were immunized with a primary series of three $10-\mu \mathrm{g}$ doses of plasma-derived HB vaccine regardless of the mother's HBV infection status. The long-term effectiveness of HB vaccine was evaluated in 2009 in Longan County. The efficacy of a plasma-derived HB vaccine use for 13-23 years and a yeast-derived recombinant $\mathrm{HB}$ vaccine for 12 years was analysed. The data showed that the efficacy of the vaccines was stable [12, 13]. As a follow-up, in order to analyse antibody persistence and protective effectiveness, we evaluated the plasma-derived HB vaccine after 22-28 years of use in this HBVendemic rural area in 2015.

\section{MATERIALS AND METHODS}

\section{Participants}

This cross-sectional study was carried out in 10 towns in Longan County in February 2015. Eligible participants were born between 1987 and 1993 and had received three $10-\mu \mathrm{g}$ doses of plasma-derived HB vaccine at ages 0,1 , and 6 months. Those who had received the first dose of vaccine more than $72 \mathrm{~h}$ after birth were excluded. The vaccines were manufactured by the Beijing Institute of Biological Products (Beijing, China). Eligible participants were identified from a database containing the records of all participants from previous cross-sectional surveys. The database included the participant's ID number, name, date of birth, dates of HBV immunization, and information identifying their parents. Individuals who had received a $\mathrm{HB}$ booster vaccination were excluded from the evaluation.

\section{Interviews and blood sample collection}

Multi-stage random sampling was used to select participants. The primary sampling unit was the village, the secondary unit was the town, and the sample size calculation was based on the HBV infection rate in Longan County [11]. About $30 \%$ of the population was sampled; the non-response rate was less than $10 \%$. Following rural community approval, trained field staff identified eligible subjects by verifying their name, date of birth, and village of residence as well as the name of the spouse, mother, or father from the study participant lists. Field workers checked the participants' immunization record booklets and recorded their demographic information, including sex, birth date, ethnicity, place of birth, and immunization history. Blood specimens $(3 \cdot 0-5 \cdot 0 \mathrm{ml})$ were collected by venepuncture, transported in a cool box during field work, and transferred to a laboratory freezer within $5 \mathrm{~h}$ of collection for storage at $-20^{\circ} \mathrm{C}$.

\section{HBV serology}

Serological testing was carried out at the Guangxi Centers for Disease Control and Prevention. Serum samples were tested for three HBV markers: HBsAg, anti-HBsAg (anti-HBs), and anti-HB core antigen (anti-HBc) using micro-particle enzyme immunoassay (MEIA) kits (AXSYM, Abbott, USA). Positivity was defined as an $\mathrm{HBsAg} \mathrm{S} / \mathrm{N} \geqslant 2 \cdot 0$, an anti-HBc $\mathrm{CO} / \mathrm{S}$ $>1 \cdot 0$, and an anti-HBs concentration of $\geqslant 10 \mathrm{mIU} / \mathrm{ml}$.

\section{Statistical analysis}

All participant information and laboratory data were entered into an SPSS v. 14.0 (SPSS Inc., USA) database and statistical analysis was carried out with SAS v. 8.0 software (SAS Institute Inc., USA). Statistical comparisons of the prevalence of HBV serological markers in men and women and across the various birth-year cohorts were performed using $\chi^{2}$ tests. The trend of change in the prevalence of HBV markers with birth year was evaluated using the $\chi^{2}$ trend test. 


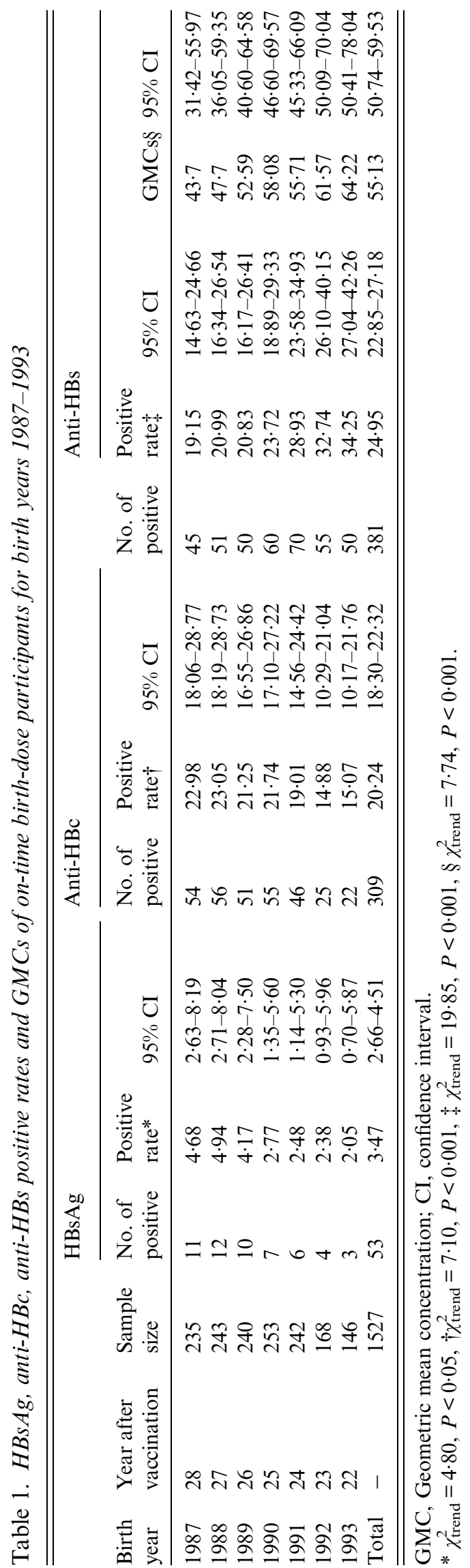

As a measure of vaccine effectiveness, we assessed long-term protection by determining HBsAg prevalence. Vaccine effectiveness was calculated as:

HBsAg prevalence in vaccinated

$$
\begin{gathered}
\text { subjects in } 2015 \\
\begin{array}{c}
\text { HBsAg prevalence in unvaccinated } \\
\text { subjects in } 1985
\end{array}
\end{gathered}
$$

All $P$ values were two-tailed and $P<0.05$ was considered to be significant. The $95 \%$ confidence intervals (CIs) for the prevalence of HBV serological markers were estimated.

\section{Ethical approval}

The study was approved by the Ethics Committee of the Chinese Center for Disease Control and Prevention. The purpose of the study and the use of the information gathered were explained to the participants by research staff. Written informed consent was obtained from each participant before enrolment and blood collection.

\section{RESULTS}

\section{Participant demographics}

A total of 1774 subjects who were born between 1987 and 1993 and had been immunized with plasmaderived HB vaccine were included in the study; 140 subjects had received a booster dose and were therefore excluded from the analysis. The remaining 1634 participants were enrolled, of whom $1072(65 \cdot 61 \%)$ were men and $562(34.39 \%)$ were women. Most of the participants $(1623,99 \cdot 33 \%)$ were of Zhuang ethnicity. The first dose of HB vaccine had been given to 1527 of the participants $(93.45 \%)$ within $24 \mathrm{~h}$ of birth and to 107 participants $(6 \cdot 55 \%)$ more than $24 \mathrm{~h}$ after birth but within $72 \mathrm{~h}$.

\section{Anti-HBs positivity and geometric mean concentration (GMC)}

The overall anti-HBs positivity rate of the 1527 participants who received the first dose within $24 \mathrm{~h}$ of birth was $24 \cdot 95 \%$ (95\% CI $22 \cdot 85-27 \cdot 18)$. The positivity rate decreased significantly annually following immunization $\left(\chi_{\text {trend }}^{2}=19.85, P<0.001\right)$. Of the 1987 birth cohort, only $19 \cdot 15 \%$ of participants were anti-HBs 


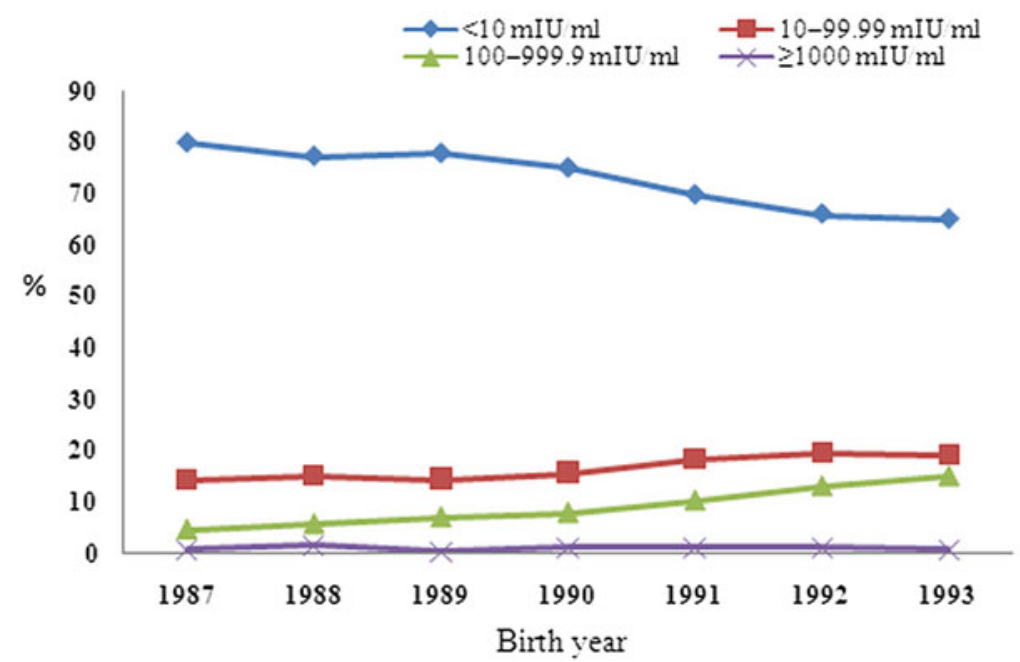

Fig. 1. Geometric mean concentration proportion of subjects for birth years 1987-1993 who received routine plasma-derived hepatitis $\mathrm{B}$ vaccine immunization with at ages 0,1 , and 6 months.

positive (Table 1). The GMC of all anti-HBs-positive participants was $55.13 \mathrm{mIU} / \mathrm{ml}$, with the value highest in the 1993 birth group $(64.22 \mathrm{mIU} / \mathrm{ml})$ and the lowest value in the 1987 birth group $(43.70 \mathrm{mIU} / \mathrm{ml})$. The anti-HBs GMC deceased significantly after immunization $\left(\chi_{\text {trend }}^{2}=7.74, \quad P<0.001\right.$; Table 1$)$. The anti-HBs-positive participants were stratified by anti-HBs concentration (Fig. 1). Most positive participants $(14 \cdot 47-16.96 \%)$ had anti-HBs concentrations of $10-99.99 \mathrm{mIU} / \mathrm{ml}$, with $4 \cdot 68-15 \cdot 07 \%$ having concentrations between 100 and $999.9 \mathrm{mIU} / \mathrm{ml}$. No more than $2 \%(0 \cdot 42-1 \cdot 65 \%, 1 \cdot 05 \%$ in total $)$ had concentrations $\geqslant 1000 \mathrm{mIU} / \mathrm{ml}$. The size of each of these groups decreased gradually following immunization from 1987 to 1993 . However, the proportion of participants with an anti-HBs concentration $<10 \mathrm{mIU} / \mathrm{ml}$ increased over time.

\section{HBsAg and anti-HBc positivity}

The overall HBsAg prevalence of the participants was $3 \cdot 79 \%(62 / 1634)$, which for participants who were administered an 'on-time' (i.e. within $24 \mathrm{~h}$ ) birth dose was $3 \cdot 47 \%$ (53/1527), while that of participants who had received a delayed first dose of vaccine was $8 \cdot 41 \%(9 / 107)\left(\chi^{2}=5 \cdot 40, P<0 \cdot 05\right)$. The HBsAg prevalence in men $(3.73 \%, 40 / 1072)$ did not differ significantly from women $\left(3.91 \%, 22 / 562, \chi^{2}=0.034, P>\right.$ $0 \cdot 05)$. The positivity rate of subjects who received the first vaccine dose within $24 \mathrm{~h}$ of birth increased by year following immunization $\left(\chi_{\text {trend }}^{2}=4 \cdot 80, P<\right.$ $0 \cdot 05$, Table 1, Fig. 2). The overall anti-HBc positivity rate of the 1527 subjects was $20 \cdot 24 \%$ (95\% CI $18 \cdot 30$ $22 \cdot 32$ ), and the rates increased by year following immunization $\left(\chi_{\text {trend }}^{2}=7 \cdot 10, \quad P<0 \cdot 001\right.$, Table 1 , Fig. 2). Correlation analysis of the HBsAg and Anti-HBs positivity rate was carried out. The data show that the positive value of HBsAg and anti-HBs were correlatively significant (correlation coefficient $=$ 0.899, $P<0 \cdot 05$, Fig. 3).

\section{Vaccine effectiveness of on-time birth-dose participants}

Compared to the $17.9 \%$ HBsAg-positive ratio reported in the 1985 survey of individuals aged $>20$ years [11], the overall protective effectiveness of the vaccine (i.e. in preventing $\mathrm{HBs} A g$ positivity) was found to be $73.91 \%$ in this study. Effectiveness declined with the number of years since immunization, being lowest in participants born in 1987 and highest in those born in $1993(73 \cdot 86,72 \cdot 40,73 \cdot 70,84 \cdot 52$, $86 \cdot 15,87 \cdot 70$, and $88 \cdot 55 \%$, respectively).

\section{HBsAg-positive rate in four previous cross-sectional surveys}

Four cross-sectional surveys have been performed, based on the population analysed in the current study. The HBsAg-positive rates observed in each survey were significantly lower than the baseline data obtained in 1985 (Fig. 4). However, in the current survey, conducted in 2015, the HBsAg-positive rates for the 1987, 1988, and 1989 birth cohorts were higher than those for 1994 and 2005 [11, 14]. There may 


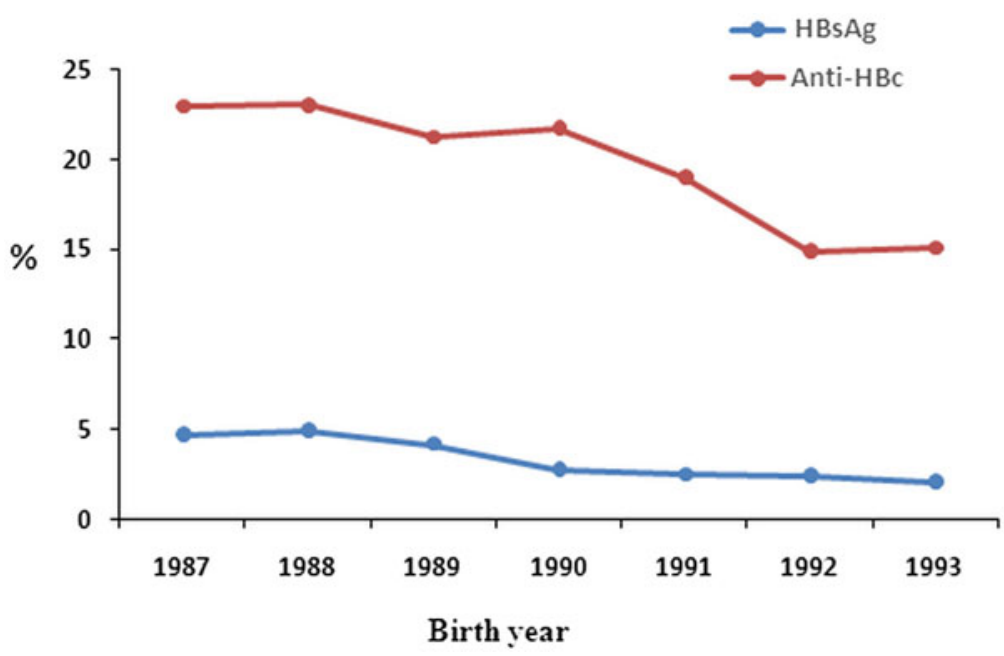

Fig. 2. HBsAg and anti-HBc positive rates of subjects for birth years 1987-1993 who received routine plasma-derived hepatitis $\mathrm{B}$ vaccine immunization at ages 0,1 , and 6 months.

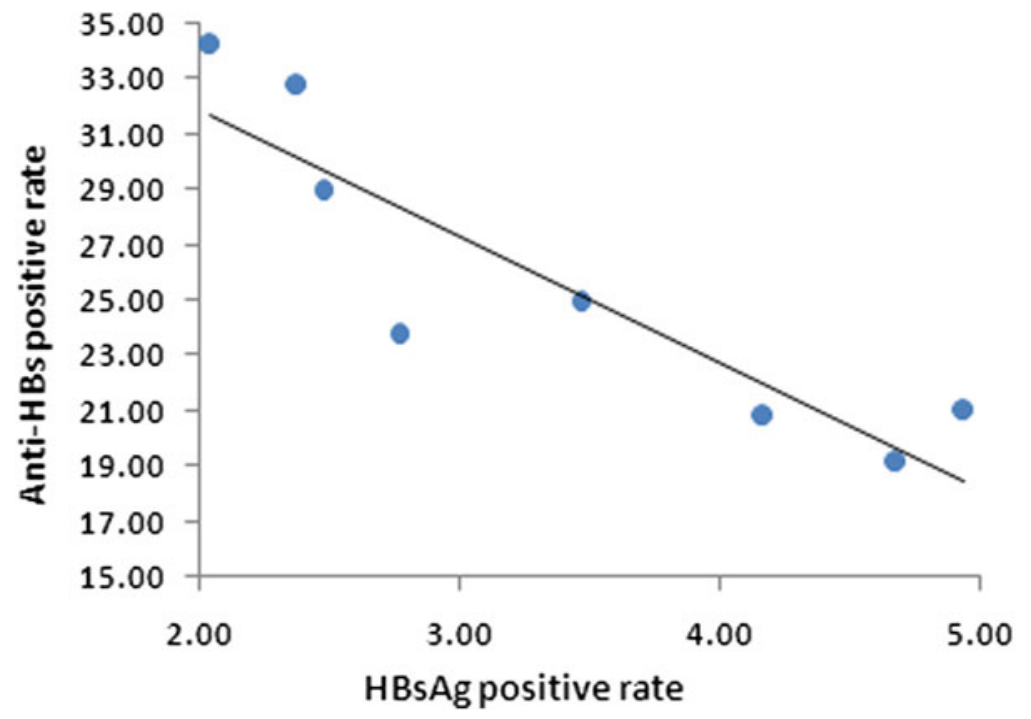

Fig. 3. Correlation analysis result of HBsAg and anti-HBs positive rate, 2015.

have been some bias in the results of the survey carried out in 2009 because of the small sample analysed, but the HBsAg-positive rates in the 1987 and 1989 birth cohorts were more than $6 \%[12,15]$.

\section{DISCUSSION}

Before evaluating the long-term effectiveness of $\mathrm{HB}$ vaccine, confounding factors such as dosage, immunization procedure, type of vaccine, and age at immunization should be taken into consideration. To ensure the current survey results were comparable with historical data, we selected participants who had received three $10-\mu \mathrm{g}$ doses of plasma-derived $\mathrm{HB}$ vaccine during infancy, using the same immunization schedule. The results demonstrate that the average HBsAg prevalence rate in the 'on-time' birth-dose group was significantly lower than that in the delayed-dose group. These findings confirm those of our previous study in Longan County $[12,13]$ and indicate that it is crucial to administer the first dose of $\mathrm{HB}$ vaccine within $24 \mathrm{~h}$ of birth in order to prevent and control HBV infection.

In this survey it was found that both the overall anti-HBs positivity rate $(24.95 \%)$ and the GMCs $(55.13 \mathrm{mIU} / \mathrm{ml})$ of all participants decreased with time after immunization. The proportion of participants with anti-HBs GMCs from 10 to $99.99 \mathrm{mIU} / \mathrm{ml}$ 


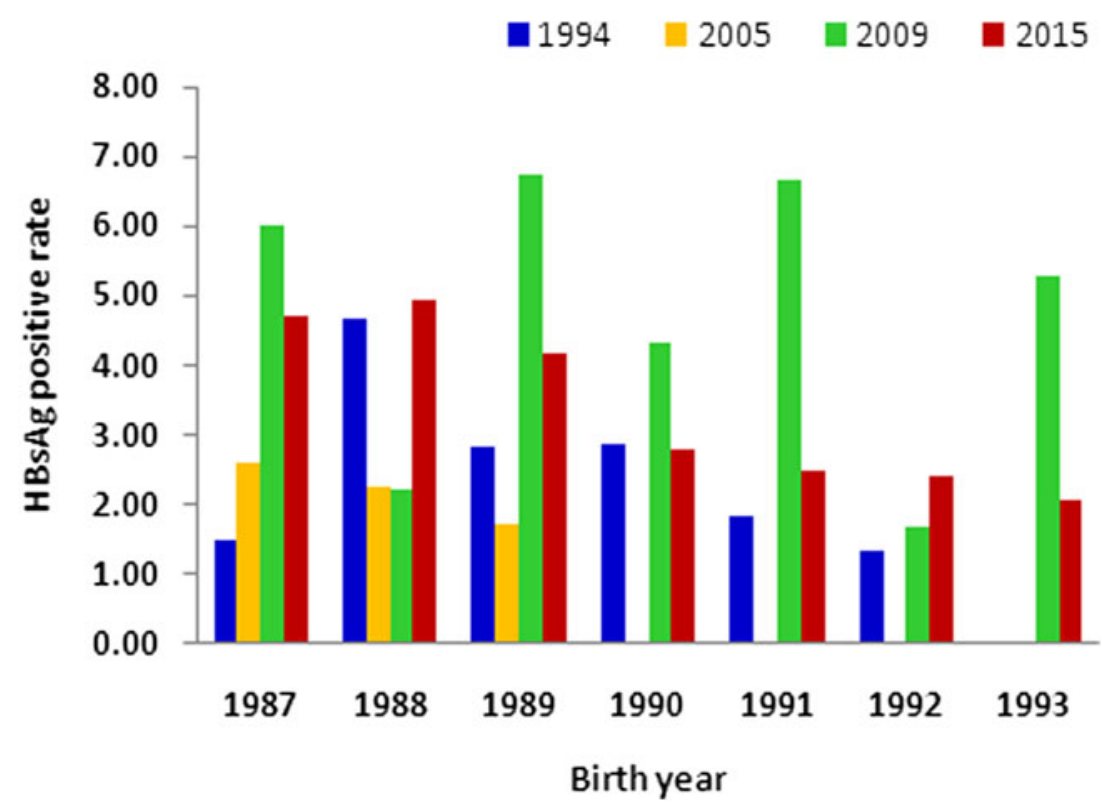

Fig. 4. Survey results of HBsAg-positive rate of 1987-1993 birth cohorts in 1994, 2005, 2009 and 2015, respectively.

and from 100 to $999.99 \mathrm{mIU} / \mathrm{ml}$ decreased in each age cohort from 1993 to 1987 . These findings are consistent with those of our study carried out in 2009 [12, 13, 15]. Interestingly, 18 participants were found to be both anti-HBs and anti-HBc positive, and 16 of these participants had antibody concentrations $\geqslant 1000 \mathrm{mIU} / \mathrm{ml}$. This may be explained by HBV natural infection (a potential natural booster), as a natural infection would have a similar effect as a vaccine booster. A study from Poland found that despite a low incidence of $\mathrm{HB}$, natural boosters such as those related to dental or surgical treatments, may stimulate post-vaccination immunity. It was also reported that HBV infection and chronic HB may occur in previously vaccinated children [16].

It was found that the HBsAg-positive rates of this survey were decreased for different birth years. The main possible reason for this phenomenon was the protective effectiveness of anti-HBs decreased by age. The HBsAg positivity rates of the 1987-1993 birth cohorts were higher than those reported in previous studies carried out in 1994 and 2005 in the same population. For participants born in 1987, HBsAg positivity was $2 \cdot 58 \%(5 / 194)$ in the 2005 survey (age 18 years) [14], and 6\% (6/100) in 2009 (age 22 years) $[13,15]$. In this survey, conducted in 2015 (age 28 years), the HBsAg positivity rate was $4.68 \%$ (11/ 235). It is suspected that the probability of HBV infection increases when the immunized population enters adulthood, i.e. aged $>18$ years. Moreover, similar to
HBsAg, the anti-HBc positivity rates were also significantly higher than previous studies. There are three possible reasons for the high $\mathrm{HBsAg}$ and anti-HBc positivity rates in Longan County. First, the failure to prevent mother-to-child transmission (PMTCT) may be a major concern, especially for newborns whose mothers have a high titre of HBV DNA. The prevalence of HBsAg in women of child-bearing age was over $15 \%$ in Longan County before the introduction of HB immunization. Administration of hepatitis B immunoglobulin (HBIG) was a crucial procedure for PMTCT [17]. However, HBIG administration was not used for immunoprophylaxis in 1987-1993 in Longan County. Moreover, it was reported that serum HBV DNA can be detected in children born to HBsAg-positive mothers even after anti-HBs antibodies are induced by HB immunization [18, 19], which suggests that children born to HBsAg-positive mothers were at risk of becoming HBV carriers even when immunoprophylaxis was successfully administered. Second, some participants may have lost immune memory 22-28 years after receiving HB immunization. Many studies have shown that although anti-HBs positivity rates and GMC decline gradually after immunization, immune memory still functions when faced with HBV invasion [20-24]. However, a survey conducted in Longan County in 2006 reported that in a group of participants who had received plasma-derived vaccine $16-18$ years previously and were anti-HBs negative, $20 \cdot 18 \%$ remained 
anti-HBs negative after a single booster dose [25]. Jan et al. reported that $24.4 \%$ of 127 college students aged 18-23 years in Taiwan who had received HB immunization were seronegative for $\mathrm{HBsAg}$, anti-HBc and anti-HBs antibodies and remained negative 1 month post-booster vaccination [26]. These studies suggest that some people lose immune memory as well as serum antibody stimulated by the HB vaccine, and the loss of protection places them at risk of HBV infection. Third, the age of the survey participants ranged from 22 to 28 years. As adults (aged $>18$ years) they may encounter numerous risks of HBV exposure and infection. These include infected household contacts, sexual contact with HBV-infected partners, unsafe injections, haemodialysis, transfusion, organ transplantation, and dental and surgical procedures [27, 28]. When children become adults, the onset of sexual activity may thus challenge their HB immunity. Therefore, it is expected that those study participants without sufficient protection from anti-HBs antibodies would be at increased risk of HBV infection in Longan County, a high HBV prevalence area.

In conclusion, we showed that the HBsAg and anti-HBc positivity rates in Longan County, a high HBV endemic area, increased 22-28 years after immunization. Anti-HBs positivity and GMCs decreased significantly in the years after vaccination, and although sampling bias may have influenced the results, the long-term effectiveness of the plasmaderived HBV vaccine also declined during this period. A booster dose may be necessary for subjects with serum anti-HBs concentrations $<10 \mathrm{mIU} / \mathrm{ml}$ who are not infected with HBV. In addition, it would be helpful to study T-cell immune memory in response to $\mathrm{HB}$ vaccine $\geqslant 22$ years after primary immunization. However, there are some limitations to this survey, such as some baseline information, including smoking, drinking, history of drug abuse being absent, the loss to follow-up rate of each stage and possible sampling bias. Further observations of the long-term protection of this plasmaderived HBV vaccine should be continued in future years.

\section{ACKNOWLEDGEMENTS}

We thank the staff of the Centers of Disease Control and Prevention of Longan County for helping coordinate the study. We also thank the village healthcare workers for their help and assistance in performing this study. This research is supported by the Chinese
National Science and Technology Major Project (grant no. 2012ZX10002-001-005-001); the National Natural Science Foundation of China (grant nos. 81360443 and 81560546); and Guangxi Natural Science Foundation (grant no. 2013GXNSFBA019194).

\section{DECLARATION OF INTEREST}

None.

\section{REFERENCES}

1. WHO. Hepatitis B fact sheet, 2015 (http://www.who.int/ mediacentre/factsheets/fs204/en/index.html).

2. Meireles LC, Marinho RT, Van Damme P. Three decades of hepatitis B control with vaccination. World Journal of Hepatology 2015; 7: 2127-2132.

3. Chang MH. Cancer prevention by vaccination against hepatitis B. Recent Results in Cancer Research 2009; 181: $85-94$.

4. Ayoola AE, et al. The decline of hepatitis B viral infection in South-Western Saudi Arabia. Saudi Medical Journal 2003; 24: 991-995.

5. Davaalkham D, et al. Impact of the universal hepatitis B immunization program in Mongolia: achievements and challenges. Journal of Epidemiology 2007; 17: 69-75.

6. Shen LP, et al. Epidemiological changes in hepatitis B prevalence in an entire population after 20 years of the universal HBV vaccination programme. Epidemiology and Infection 2011; 139: 1159-1165.

7. Liang $\mathbf{X}$, et al. Evaluation of the impact of hepatitis B vaccination among children born during 1992-2005 in China. Journal of Infectious Diseases 2009; 200: 39-47.

8. Liang $\mathbf{X}$, et al. Epidemiological serosurvey of hepatitis B in China - declining HBV prevalence due to hepatitis B vaccination. Vaccine 2009; 27: 6550-6557.

9. Ding ZR. Distribution of viral hepatitis B infection in Guangsi Province [in Chinese]. Zhonghua liu xing bing xиe za zhi 1982; 3: 84-87.

10. Fang ZL, et al. Prevalence of hepatitis B virus infection in a highly endemic area of southern China after catch-up immunization. Journal of Medical Virology 2012; 84: 878-884.

11. Li RC, et al. The effect of hapatitis B vaccination on epidemiology of hepatitis B virus [in Chinese]. Chinese Journal of Vaccines and Immunization 1996; 2: 56-60.

12. Wang F, et al. The long-term efficacy, 13-23 years, of a plasma-derived hepatitis B vaccine in highly endemic areas in China. Vaccine 2015; 33: 2704-2709.

13. Shen LP, et al. Efficacy of yeast-derived recombinant hepatitis B vaccine after being used for 12 years in highly endemic areas in China. Vaccine 2012; 30: 6623-6627.

14. Shen LP, et al. Evaluation of long-term protective efficacy of infant hepatitis Plasma-derived vaccine immunization [in Chinese]. Practical Prevention Medicine 2006; 13: 1425-1427.

15. Shen LP, et al. Study on long-term efficacy and duration of hepatitis B vaccine in China [in Chinese]. 
Chinese Journal of Microbiology and Immunology 2013;33: 6-10.

16. Pokorska-Lis M, Marczynska M. Influence of natural boosters on long-term immunity against hepatitis B. Medycyna Wieku Rozwojowego 2011; 15: 62-68.

17. Kang W, et al. Risk factors associated with immunoprophylaxis failure against mother to child transmission of hepatitis B virus and hepatitis B vaccination status in Yunnan province, China. Vaccine 2014; 32: 3362-3366.

18. Matsumoto T, et al. Efficacy of immunization of highrisk infants against hepatitis B virus evaluated by polymerase chain reaction. Journal of Medical Virology 1997; 53: 255-260.

19. Kato H, et al. Long-term efficacy of immunization against hepatitis B virus in infants at high-risk analyzed by polymerase chain reaction. Vaccine 1999; 18: 581-587.

20. Mendy M, et al. Observational study of vaccine efficacy 24 years after the start of hepatitis B vaccination in two Gambian villages: no need for a booster dose. PLoS ONE 2013; 8: e58029.

21. Dentinger CM, et al. Persistence of antibody to hepatitis $\mathrm{B}$ and protection from disease among Alaska natives immunized at birth. Pediatric Infectious Disease Journal 2005; 24: 786-792.

22. Yazdanpanah B, Safari M, Yazdanpanah S. Persistence of HBV vaccine's protection and response to hepatitis
B booster immunization in 5- to 7-year-old children in Kohgiloyeh and Boyerahmad Province, Iran. Hepatitis Monthly 2010; 10: 17-21.

23. Bagheri-Jamebozorgi M, et al. The persistence of anti-HBs antibody and anamnestic response 20 years after primary vaccination with recombinant hepatitis $\mathrm{B}$ vaccine at infancy. Human Vaccines \& Immunotherapeutics 2014; 10: 3731-3736.

24. Poovorawan Y, et al. Persistence and immune memory to hepatitis $B$ vaccine 20 years after primary vaccination of Thai infants, born to $\mathrm{HBsAg}$ and $\mathrm{HBeAg}$ positive mothers. Human Vaccines \& Immunotherapeutics 2012; 8: 896-904.

25. Li YP, et al. Studies on the status of immune memory after completion of hepatitis B vaccination [in Chinese]. Zhonghua liu xing bing xue za zhi 2007; 28: 319-321.

26. Jan CF, et al. Determination of immune memory to hepatitis B vaccination through early booster response in college students. Hepatology 2010; 51: 1547-1554.

27. Ozer A, et al. Risk factors of hepatitis B virus infection in Turkey: a population-based, case-control study: risk factors for HBV infection. Hepatitis Monthly 2011; 11: 263-268.

28. Janahi EM. Prevalence and risk factors of hepatitis B virus infection in Bahrain, 2000 through 2010. PLoS ONE 2014; 9: e87599. 